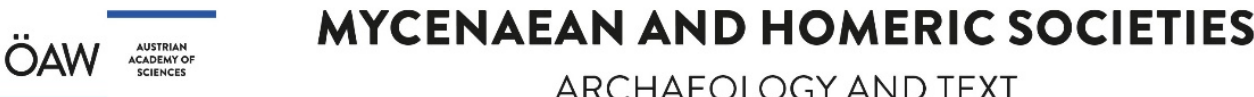 \\ ARCHAEOLOGY AND TEXT
}

Collected Papers of

\section{SIGRID DEGER-JALKOTZY}

edited by Birgitta Eder and Ulrike Schuh

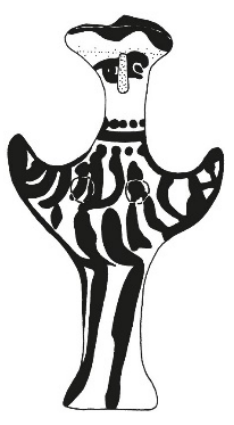

08.

S. Deger-Jalkotzy

Das Problem der „Handmade Burnished Ware“ von Myk. IIIC

in: S. Deger-Jalkotzy (ed.), Griechenland, die Ägäis und die Levante während der Dark Ages vom 12. bis zum 9. Jh. v.Chr. Akten des Symposion von Stift Zwettl (NÖ), 11.-14. Oktober 1980, Veröffentlichungen der Kommission für mykenische Forschung $10=$ Sitzungsberichte der Österreichischen Akademie der Wissenschaften, philosophisch-historische Klasse 418 (Vienna 1983) 161-168

(C) Verlag der ÖAW

mit freundlicher Genehmigung / with kind permission

Dieses Dokument darf ausschließlich für wissenschaftliche Zwecke genutzt werden (Lizenz CC BY-NC-ND), gewerbliche Nutzung wird urheberrechtlich verfolgt. 

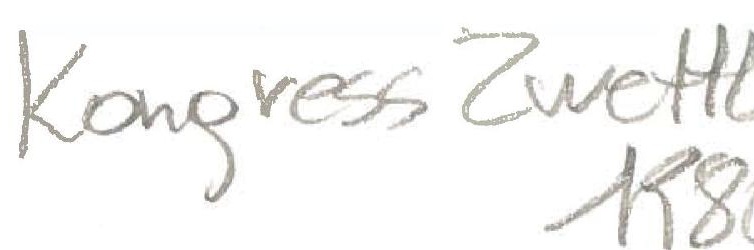

ÖSTERREICHISCHE AKADEMIE DER WISSENSCHAFTEN PHILOSOPHISCH-HISTORISCHE KLASSE

SITZUNGSBERICHTE, 418. BAND

\section{VERÖFFENTLICHUNGEN}

DER KOMMISSION FUR MYKENISCHE FORSCHUNG

BAND 10

\section{GRIECHENLAND, DIE ÄGÄIS UND DIE LEVANTE WÄHREND DER „DARK AGES“ VOM 12. BIS ZUM 9.JH. V.CHR.}

Akten des Symposions von Stift Zwettl (NÖ)

$$
\text { 11.-14. Oktober } 1980
$$

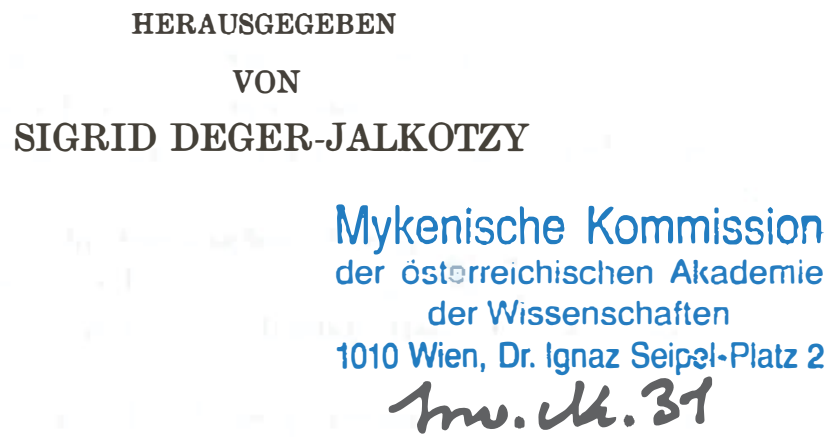

Mykenische Kommission der östorreichischen Akademie der Wissenschaften

Imo.ck.31

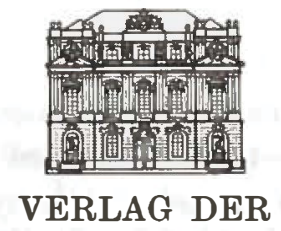

ÖSTERREICHISCHEN AKADEMIE DER WISSENSCHAFTEN 
Vorgelegt von w. M. FRITZ SCHACHERMEYR in der Sitzung am 24. Juni 1981

rE.3k.unt

Alle Rechte vorbehalten

一ISBN 3700105967

Copyright (C) 1983 by

Österreichische Akademie der Wissenschaften

Wien

Druck: Ernst Becvar, A-1150 Wien 


\section{INHALTSVERZEICHNIS}

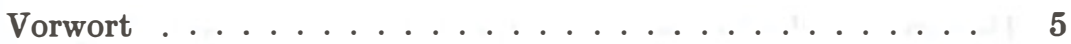

Begrüßung durch Ernst Kirsten $\ldots \ldots \ldots \ldots \ldots$

H. Отten (Mainz): Die letzte Phase des Hethitischen Großreiches nach den Texten . . . . . . . . . . . . . 13

Diskussion (Schachermeyr, Lehmann, Otten) . . . . . . 21

K. Bittel (Heidenheim): Die archäologische Situation in Kleinasien um 1200 v. Chr. und während der nachfolgenden vier Jahrhunderte

Diskussion (Akurgal, Bittel, Jalkotzy, Otten, Lehmann, Do-

besch, Hölbl, Schachermeyr, Kirsten, Dothan)

E. AKurgal (Ankara): Das Dunkle Zeitalter Kleinasiens . . . .

G. A. LehmanN (Köln): Zum Auftreten von „Seevölker“-Gruppen im östlichen Mittelmeerraum - eine Zwischenbilanz

Diskussion (Schachermeyr, Kirsten, Dothan, Bittel, Lehmann, Jalkotzy)

T. Dothan (Jerusalem): Some Aspects of the Appearance of the Sea Peoples and Philistines in Canaan . . . . . . . . .

Diskussion (Schachermeyr, Jalkotzy, Dothan, Hölbl, Lehmann)

G. Holbl (Wien): Die historischen Aussagen der ägyptischen Seevölkerinschriften

Diskussion (Schachermeyr, Lehmann, Hölbl, Dobesch, Akurgal)

P. G. Themelis (Athen): Die Nekropolen von Lefkandi-Nord auf Euboea

Diskussion (Schachermeyr, Themelis, Georgiev, Eibner, Hölbl)

S. Deger-Jalkotzy (Wien): Das Problem der „Handmade Burnished Ware"

Diskussion (Bittel, Jalkotzy, Schachermeyr, Themelis, Georgiev, Eibner, Kirsten, Hölbl, Lehmann) . . . . . . . 169

G. Dobesch (Wien): Historische Fragestellungen in der Urgeschichte 
Diskussion (Schachermeyr, Jalkotzy, Lehmann, Dobesch, Panagl, Hölbl)

F. Schachermeyr (Wien): Die Zeit der Wanderungen im Spiegel ihrer Keramik

Diskussion (Themelis, Schachermeyr, Jalkotzy, Akurgal, Eibner)

G.I.Georgiev (Sofia): Das Anfangsstadium der Früheisenzeit (12.-9. Jh. v. u.Z.) in Südost- und Nordostbulgarien . . . . 259 Diskussion (Schachermeyr, Akurgal, Jalkotzy, Georgiev) . 269

J. BouzeK (Prag): Der Vardar- und Morava-Bereich in seinem Verhältnis zu Griechenland zwischen 1200 und 900 v. u. Z.

C. EIBNer (Wien): Der Bereich der Mittleren Donau während der späten Bronze- und frühen Eisenzeit

Diskussion (Lehmann, Eibner, Jalkotzy, Schachermeyr, Pa-

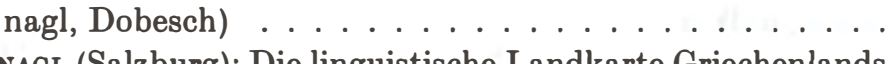

O. Panagl (Salzburg): Die linguistische Landkarte Griechenlands während der Dunklen Jahrhunderte

Diskussion (Schachermeyr, Panagl, Lehmann, Jalkotzy, Eibner)

E.KIRSTEN (Wien): Gebirghirtentum und Seßhaftigkeit - die

Bedeutung der Dark Ages für die griechische Staatenwelt:

Doris und Sparta

Diskussion (Schachermeyr, Jalkotzy)

Nachtrag

Ansprache von Kurt BITTEL

Schlußwort von Fritz SchacherMEyR

Abkürzungsverzeichnis

Sachwörterverzeichnis 


\section{DAS PROBLEM DER „HANDMADE BURNISHED WARE“ VON MYK. III C}

\section{Sigrid Deger-JALKotZy (Wien)}

Ein großer Teil meines Zwettler Vortrages war dem Referat jener Tatbestände und Probleme gewidmet, die ich schon zuvor monographisch dargestellt hatte ${ }^{1}$. Ich kann mich daher hier, in der gedruckten Fassung des Vortrages, wesentlich kürzer halten und ergänzend neue Fakten anfügen bzw. einzelne Probleme nochmals hervorheben, vor allem jene, die offenbar Anlaß zu Mißverständnissen gegeben haben.

Die jährlichen Kampagnen in Aigeira haben das in meiner Veröffentlichung von 1977 entworfene Bild von der „Handmade Burnished Ware $\left(=\mathrm{HBW}\right.$ )" dieses Fundortes nicht wesentlich verändert ${ }^{2} . \mathrm{Zu}$ korrigieren habe ich allerdings meine seinerzeitige Angabe, daß das betreffende Material nur in der ersten Siedlungsphase des Frühen SH IIIC in Aigeira, zusammen mit mykenischer Ware, aufgetreten sei ${ }^{3}$. Neue Befunde haben ergeben, daß beide Siedlungsphasen des Frühen IIIC in Aigeira mykenische und nicht-mykenische Keramik im Verband enthielten ${ }^{4}$. Dies steht nun durchaus im Einklang mit allem, was wir von den anderen Fundplätzen dieser Keramik in Griechenland wissen. Für ein Fortleben der handgemachten Fremdware ins Mittlere SH III C bestehen in Aigeira weiterhin keine Anhaltspunkte ${ }^{5}$.

[Korrekturzusatz: Dagegen zeigt K. KILIAN im neuesten Tirynsbericht Funde von HBW sogar aus späten SH III C-Kontexten an, vgl. AA 1982, 399.]

${ }^{1}$ S. DEger-JALKotzy, Fremde Zuwanderer im spätmykenischen Griechenland. Zu einer Gruppe handgemachter Keramik aus den Myk. IIIC Siedlungsschichten von Aigeira (Wien 1977). Hinfort: Fremde Zuwanderer.

${ }^{2}$ Abermals habe ich dem Ausgräber von Aigeira, Prof. W. Alzinger, zu danken für die Erlaubnis, das Material aus diesen Ausgrabungen verwenden und veröffentlichen zu dürfen.

3 Fremde Zuwanderer, 1.5.1.

${ }^{4}$ Der auf unserer Abb. 1 a wiedergegebene Krug datiert beispielsweise aus der jüngeren der beiden Siedlungsphasen des Frühen III C.

${ }^{5} \mathrm{Zu}$ den in meinen Fremden Zuwanderern zusammengefaßten Materialien aus griechischen Fundorten sind jene vom Menelaion getreten (BSA 76 [1981] $7 \mathrm{lff}$.), Funde aus Chania (s. u.) sowie Funde aus Kalapodi. 
Aus Tiryns werden nunmehr Funde der handgemachten Fremdware schon aus SH III B 2 gemeldet ${ }^{6}$. Ähnlich berichtet Birgitta Hallager über das Vorkommen analogen Materials bei den Ausgrabungen in Chania/Westkreta, und zwar (vereinzelt) schon im frühen 13. Jh., zur Hauptsache aber im späten 13. und im frühen 12. Jh. ${ }^{7}$. Hier freilich wird man die Publikation des Materials und seines Kontextes abwarten müssen.

Bedeutsam an diesem neuen, einschlägigen Fundzuwachs aus Chania erscheint allerdings die Existenz zweier Gattungen von Fremdkeramik nebeneinander: einerseits eine handgemachte Grobkeramik, deren Hauptformen die carinierte Tasse mit hochgezogenem Bandhenkel, sowie ein anscheinend amphorenartiges Gefäß (,,deep jar with two vertical handles") sind. Diese Gattung kennt plastische Leisten, mit und ohne Verzierung. Die andere, ebenfalls unterbrandige und aus der minoisch-mykenischen Tradition herausfallende Gattung zeichnet sich durch dunkelgraue Färbung aus - ist aber scheibengedreht! Ihr Formengut umfaßt Kylikes, Tassen, Schalen. Carinierte Tassen haben einen über den Gefäßrand hochgezogenen Stab- oder auch Bandhenkel. Diese Ware ist stets unverziert. Sie kommt nur im späten 13. und im frühen 12. Jh. vor, während die Grobware, dem Bericht Frau Hallagers nach, auch schon im frühen 13. Jh. auftrat (s. oben).

Zur Charakteristik und zur Herstellungsweise der handgemachten Fremdkeramik haben sich J.B. Rutter ${ }^{8}$, K. Kilian ${ }^{9}$ und ich selbst ${ }^{10}$ geäußert. Darnach steht es außer Frage, daß es sich um eine nichtmykenische, fremde Keramikgattung handelt. Wie wenig Gisela Walbergs Einwand, daß auch die mykenische Tradition handgemachtes Geschirr kannte ${ }^{11}$, im Zusammenhang mit der hier behandelten Ware gelten kann, zeigt vollends nun die jüngst von Miss Mountjoy vorgelegte, genuin mykenische handgemachte Keramik unter dem Material vierer mykenischer Brunnen aus Athen ${ }^{12}$. Im Gegensatz zur mykeni-

${ }^{6}$ K. KILIAN, AA 1981, 181.

7 Birgitta Pálsson Hallager, A new social class in Late Bronze Age Crete. Vortrag beim Colloqiuum on Minoan Society 1981 in Cambridge (im Druck).

${ }^{8}$ J. B. RUTTER, AJA 79 (1975) $17 \mathrm{ff}$.

${ }^{9}$ K. KILIAN, in: Atti XX Riunione Scientifica in Basilicata 1976 (1978) $311 \mathrm{ff}$.

${ }^{10}$ Fremde Zuwanderer, Kapitel 2.

$"$ G. WALBERG, AJA 80 (1976) $186 \mathrm{f}$.

12 P. A. MountJoy, Four Early Mycenaean Wells from the South Slope of the Acropolis at Athens (Gent 1981): Fig. 23, 284; 24, 309; 25, 345.356; Tfl. 24a, 345; Fig. 31, 419; Tfl. 27b, 419. 
schen handgemachten Ware ist es also legitim, von handgemachter Fremdkeramik (als Äquivalent zu „Handmade Burnished Ware“) zu sprechen.

Die Frage nach einem mykenischen oder nicht-mykenischen Stammbaum dieser Keramik sollte demnach kein Problem mehr sein.

Ebenso hat nun auch $\mathrm{K}$. Kilian betont, daß angesichts des Formeninventars dieser Fremdware nicht länger von Importbehältern gesprochen werden kann ${ }^{13}$. Skeptischen Äußerungen über mangelnde Quantitäten der entsprechenden Keramikfunde ${ }^{14}$ möchte ich noch einmal den in Hunderte gehenden Fragmentbestand - neben einigen Ganzgefäßen und einer Reihe von Großfragmenten, die Aufschluß über die Gefäßformen geben (wie viele Ganzgefäße erwartet man eigentlich aus einer Siedlungsgrabung?) — aus Aigeira verweisen, ferner auf die große Anzahl von Fragmenten, die Frau Hallager nennt ${ }^{15}$, und schließlich auch auf Beispiele von Spinnwirteln, die in dieser Machart verfertigt sind (Abb. 1 c). Vor allem bei den Spinnwirteln ${ }^{16}$ liegt der Verdacht auf Import wohl nicht sehr nahe. Nein, die Töpferwaren, die der handgemachten, fremden Keramik zuzuzählen sind, wurden in Griechenland selbst erzeugt: mykenischen Griechen freilich wird man ihre Produktion kaum zuschreiben ${ }^{17}$.

Somit erheben sich in erster Linie zwei Hauptprobleme im Zusammenhang mit der hier diskutierten Keramik:

13 AA 1981, 181.

14 Rez. Fremde Zuwanderer durch Susan SHERRATT, JHS 99 (1979) 200.

15 Hallager, a. Anm. 7 a. $O$.

16 Spinnwirteln in handgemachter Fremdware fanden sich, der freundlichen Mitteilung von Frau Helen Hughes-Brock zufolge, auch in Mykene. Frau Elizabeth FrENCH danke ich für die Auskunft, daß es sich um Stücke aus Kontexten des fortgeschrittenen SH III C handelt (vgl. dazu auch u. S. 445), sowie für die Erlaubnis zur Erwähnung dieses Materials.

17 Ähnlich K. A. WARDLE, Godišnjak Sarajevo 15 (1977) 188f. - Nancy SANDARs, The Sea Peoples (London 1978) $191 \mathrm{f}$. bietet einen etwas befremdlichen Beitrag zur Genese der handgemachten Fremdware: nach der Katastrophe von SH III B hätte es keine mykenischen Töpferwaren zu kaufen gegeben. Mykenische Hausfrauen hätten also handgemachte Ware im Meilerbrand hergestellt, bzw. sei eine neue soziale Schichte von Emporkömmlingen nicht interessiert an guter Keramik gewesen. - Nun sind „vorübergehende" Rückfälle in prähistorische Zustände, auch nach den größten Katastrophen, in der Geschichte recht selten bis nicht belegt. Außerdem produzierte das Frühe SH III C sehr wohl mykenische Keramik, wenn auch nicht noble Ware (nach der Katastrophe hatte man wohl wirklich andere Probleme, als neue Keramikstile zu schaffen!), sondern einfaches Alltagsgeschirr. Beweis dafür ist der Töpferofen, der in Aigeira im Frühen III C errichtet wurde. 
1. Woher kamen ihre Hersteller, d.h. in welchen geographischen Bereich läßt sich diese Keramik zurückverfolgen?

2. Können die Hersteller der handgemachten Fremdkeramik mit den Zerstörungen der mykenischen Paläste am Ende des 13.Jh. v. Chr. in Beziehung gebracht werden?

Was das erste Problem betrifft, so stehen sich im wesentlichen drei Meinungen gegenüber. Einem Ursprung aus Südost-Europa sprach J. B. Rutter das Wort ${ }^{18}$. Dieser Vorstellung entspricht die Coarse Ware von Troia VIIb1, aber auch ein Teil der in Griechenland gefundenen handgemachten Fremdware, wobei die Chronologie in beiden Gebieten parallel läuft ${ }^{19}$.

Eine Schwierigkeit für diese These liegt aber primär darin, daß die Parallelen zwischen dem Material aus Troia und jenem aus Griechenland nur partiell sind. M. Popham und Elizabeth Milburn sahen für ihr Material aus Lefkandi viel eher Konnexe mit Unteritalien ${ }^{20}$. Ähnliches gilt für die handgemachte Fremdkeramik von Aigeira. Doch habe ich ausdrücklich die Gebiete diesseits und jenseits der Adria als unmittelbare Herleitungszone des Großteiles des Aigeiratischen Materials bezeichnet $^{21}$ (s. auch weiter unten). - Frau Hallager bringt ebenfalls das neue Material aus Kreta mit dem südadriatischen Raum in Verbindung, hat aber wiederum eher Süditalien als die dalmatinische und illyrische Seite der Adria im Auge, womit sie konform mit Popham und Milburn geht ${ }^{22}$.

K. Kilian dagegen schränkt für sein Tirynther Material die südadriatischen Rückverbindungen auf Nordwestgriechenland ein ${ }^{23}$, glücklicherweise ohne den unpassenden Terminus „Dorierware“ weiter zu strapazieren ${ }^{24}$. Er nennt sie neutral „Nordwestgriechische Keramik“.

${ }^{18}$ RUTTER, a. Anm. 8 a. O.; AJA 80 (1976) 187 f.; vgl. auch E. FrENCHJ.B. RUTTER, AJA 81 (1977) 111 f.

19 FRENCH--RUTter, a. $O$.

${ }^{20}$ M. R. Popham-E. Milburn, BSA 66 (1971) 338.

${ }^{21}$ Fremde Zuwanderer, 4.10, 6.2019.

${ }^{22}$ Hallager, $a$. Anm. 7 a.O.

${ }^{23}$ Kilian, a. Anm. 9 a. O., sowie AA 1981, 181. Auch AA 1979, Abb.31, 3.5.6.

${ }^{24}$ Die Bezeichnung „Dorierkeramik“ bezieht sich auf die griechischen Traditionen über die Dorische Wanderung und die Dorier, die sich bekanntlich im archäologischen Befund nicht näher definieren lassen. Wenn man also einen Begriff aus der literarischen Uberlieferung der Griechen nimmt, dann muß man sich auch konsequent an diese Uberlieferung halten: für den ersten Versuch der Dorier, im mykenischen Bereich Fuß zu fassen (vgl. Herod. IX 26; Paus. VIII $5,1)$, ist die Laufzeit der handgemachten Fremdware viel zu lang. Und zur 
Demgegenüber möchte ich aber festhalten, daß wesentliche Komponenten der Fremdkeramik in Griechenland, wie etwa die carinierte Tasse (vgl. u. Abb. 2b, 2c links) ${ }^{24 a}$, oder die in meinen Fremden $\mathbf{Z} u$ wanderern (Tfl. I: 4, 6; II: 2; Farbtfl.: 4, 5) sowie die übrigen hier auf Abb. 1 und 2 vorgestellten Beispiele keine Parallelen in NW-Griechenland haben. Ähnlich argumentiert Frau Hallager für Chania ${ }^{25}$. Julia Vokotopoulou ist darüber hinaus der Ansicht, daß die in NW-Griechenland auftretenden Parallelen zur handgemachten Fremdkeramik aus dem mykenischen Bereich keine einheimische Tradition haben und nicht vor dem ausgehenden 13. Jh. datiert werden können ${ }^{26}$. Demnach wäre NW-Griechenland selbst zum Einzugsbereich der in der südlichen Adria agierenden ethnischen Gruppen erst geworden, und zwar zur gleichen Zeit wie die mykenischen Gebiete. Ich bezweifle sehr, ob man NW-Griechenland im späten 13./frühen 12. Jh. aus dem Kulturverband der südlichen Adria und des Balkans im Bereich des heutigen Jugoslavien wird trennen dürfen. Allerdings muß ich einräumen, daß das Material von Tiryns so wie jenes aus Epirus in seinem Formenbestand in der Tat Unterschiede und Limitierungen gegenüber den Beispielen aus Korakou, Aigeira oder nun auch Chania aufweist.

Der Formenbestand der an den diversen Fundorten Griechenlands zutage getretenen handgemachten Fremdware ist also keinesfalls einheitlich. Selbst an ein und demselben Fundplatz wie Aigeira konnte ich Affinitäten nach verschiedenen Richtungen hin feststellen ${ }^{27}$. Aus genau diesem Grund versuchte ich, alle diese Phänomene unter einem einheitlichen Gesichtspunkt zu verstehen, d. h. in dem Sinn, daß keine der oben referierten Standpunkte eo ipso falsch sein muß. Fiel mir doch auf, daß in allen Regionen, an denen Parallelen zu unserer Fremdware aufgetreten sind (Unteritalien, Balkan, SO-Europa ${ }^{28}$, Troia), diese

Rückkehr der Herakliden paßt sie erst recht nicht, da sie absorbiert wird im Rahmen einer Kultur, die sich nach wie vor als mykenisch begreift, mindestens bis zum Ende des 12.Jh. - Zum Dorierproblem vgl. neuerdings F. SCHACH ERMEYR, Ägäische Frühzeit IV (Wien 1980) $403 \mathrm{ff}$.

24a Zur mykenischen Tasse FS 240 als UUbernahme aus dem Fremdkeramik-Repertoire habe ich mich in der Festschrift Hedwig Kenner geäußert (Pro Arte Antiqua [Wien 1982] 54ff.).

${ }^{25}$ A. Anm.7 a.O.

${ }^{26}$ Julia Vокотороulou, Ephem. 1969, 189f. und 203, sowie brieflich (14.12.1981). - [Zu Epirus vgl. auch die anschließende Diskussion, unten S. $171 \mathrm{ff}$.

27 Fremde Zuwanderer, zusammenfassend 6.22.1-3.

${ }^{28}$ Bezüglich der Riefenware aus Makedonien haben sich neuerdings Vorschläge zu einem tieferen chronologischen Ansatz ergeben, aufgrund der 
Parallelen nicht allein aus den einheimischen Traditionen erklärbar sind. Vielmehr definieren sich die betreffenden Kulturhorizonte jeweils dadurch, daß neue Phänomene des kulturellen Habitus zu den einheimischen Traditionen getreten und mit ihnen verschmolzen sind. Sie datieren außerdem synchron mit den Funden aus dem mykenischen Bereich. Geht man nun den Ursprüngen der jeweils neuen Phänomene nach, so bieten sich $\mathrm{m}$. E. am ehesten Rückverbindungen aller dieser Facies nach dem mittleren Donauraum an ${ }^{29}$.

Ich möchte aber nochmals betonen, da ich gelegentlich offenbar falsch verstanden werde: die unmittelbaren Rückverbindungen der handgemachten Fremdware im mykenisch-griechischen Bereich gehen m. E. hauptsächlich in den adriatischen Raum. Das weitere Hinterfragen (das dem Historiker wohl ansteht) zielt nach dem Problem, wie und woher sich dort die entsprechenden Phänomene konstituierten, da eine autochthone Erklärung nun einmal nicht dafür ausreicht (vgl. auch u. Anm. 33); ebenso erhebt sich die Frage, wie die Affinitäten dieser Materialien zu den anderen oben genannten Regionen zu erklären seien, die doch immerhin einen beachtlichen geographischen Raum umfassen.

Hier nun bietet sich der Utbergang zum zweiten großen Problem in Verbindung mit der handgemachten Fremdkeramik, nämlich ob man sie im Zusammenhang sehen kann mit den Katastrophen der mykenischen Paläste am Ende des 13. Jh. Dazu ist meine Antwort schlankweg: ja. Das von Nancy Sandars entworfene Bild ${ }^{30}$, wonach die Völkerschaften des „High Barbary“ wirtschaftlich und kulturell expandiert hätten, um dann am Rande der Ägäis, gleichsam als unbeteiligte Zuschauer, zu betrachten, wie die Zivilisationen des östlichen Mittelmeeres von selbst in sich zusammenfielen, begeistert den Historiker wenig.

Befunde von Kastanas und Assiros (vgl. K. A. WARDle, BSA 75 [1980] 262). Der pseudo-kannelierte, mit Barbotine verzierte Henkel von Aigeira (Fremde Zuwanderer, 2.10 und 5.12-13) wäre darnach erheblich älter als die makedonischen Exemplare. Bevor wir nun aber unseren Henkel aus Aigeira mit den zeitlich näher, aber geographisch ferner liegenden tordierten Henkel von Očkov und Čaka direkt verbinden, wäre eine genauere Durcharbeitung der chronologischen Situation der makedonischen, aber auch der albanischen Materialien notwendig.

${ }^{29} \mathrm{Vgl}$. auch J.BOUzEK, zuletzt in diesem Band S.271ff.; zu dem als "Urnenfelderkultur" bezeichneten Kulturhabitus dieses Raumes zur gegenständlichen Zeit vgl. den Beitrag von C. EIBNER, unten S. $285 \mathrm{ff}$.

${ }^{30}$ SANDARS, a. Anm. 17 a. O., $81 \mathrm{ff}$. 
Doch hat Frau Sandars zweifellos recht, wenn sie die vordergründig-primitive Idee einer Massenbewegung „,wilder, haarflatternder Barbaren" aus Binneneuropa in die Ägäis perhorresziert. Demgegenüber versuchte ich eine modifizierte, der modernen Forschung gemäße Sicht vorzulegen von der Aktivität kleinerer, höchst dynamischer Gruppen (nicht selten das Ventil einer übervölkerten Region ${ }^{31}$ ), die genügen können, um für den Hochkulturraum auf Dauer von der verheerendsten Folge zu sein ${ }^{32}$. Bei guter Gelegenheit kann die konzentrierte Aktion solcher Verbände dazu führen, den schon angeschlagenen und geschädigten Hochkulturbereich endgültig niederzuringen: Der Vortrag von G. Dobesch wird uns dazu anschließend ausgiebiges Vergleichsmaterial aus der antiken Geschichte vorlegen. Ich neige also nach wie vor der Idee zu, daß Gruppen aus dem adriatischen Raum, vor allem aus dem Bereich der Subapenninischen Kultur ${ }^{33}$, Angriffe gegen die mykenische und ägäische (Kreta!) Welt richteten, eine Welt, die sie durch Handelskontakte, aber auch durch die Aktivitäten als Söldner ${ }^{34}$ sicherlich gut kannten ${ }^{35}$. Meine schon früher geäußerte Vermutung, daß die mykenischen Staaten mitunter bereits die Ansiedlung solcher fremder Elemente hinnehmen mußten, um weitere Angriffe zu vermeiden ${ }^{36}$, könnte durch das vereinzelte Auftreten der handgemachten Fremdkeramik schon in SH III B Evidenz erhalten haben. Utber die dunkelgraue, scheibengedrehte Fremdkeramik von Kreta möchte ich mich freilich nicht äußern, bevor ich sie zumindest im Bild gesehen habe. Ist sie ein Versuch, die Fremdware auf minoisch-mykenischen Standard zu bringen?

Welche Vorgänge schließlich der mykenischen Palastkultur tatsächlich das Ende bereiteten, wissen wir nicht. Diejenigen, die die

${ }^{31}$ Dies hat auch Frau SANDARS gesehen, vgl. a. $O ., 83$.

${ }^{32}$ Dazu vgl. Fremde Zuwanderer, 6.8.2 2 . Die andere Form von Wanderbewegungen, das langsame Vordringen größerer Gruppen in benachbarte Gebiete, habe ich ebda., 6.9.3 dargestellt. Diese Form kommt für die Vorgänge in der südlichen Adria und in der Ägäis weniger in Betracht.

${ }^{33} \mathrm{Zu}$ meiner Ansicht über die Herausbildung der Subapenninischen Kultur, wie ich sie in Fremde Zuwanderer, 6.10.4ff. dargestellt habe, stehe ich, pace Kilian (AA 1981, 181), ebenfalls. Doch soll uns dieser Punkt in der gegenwärtigen Erörterung nicht direkt beschäftigen.

${ }^{34}$ Dazu auch Frau SANDARS, a. Anm. 17 a. O., $91 \mathrm{ff}$.

${ }^{35} \mathrm{Zu}$ den in Fremde Zuwanderer, 6.11.2 ff. zusammengestellten Argumenten vgl. in diesem Band J.BOUZEKs Beitrag, zusätzlich zu seinen früheren Publikationen.

${ }^{36}$ Verf., E-QE-TA (Wien 1978) 35f. - S. auch in diesem Band G. DOBESCH für Vergleichsmaterial, unten S. 179ff. 
Beteiligung fremder Angreifer an diesen Ereignissen ausschließen und eher an innermykenische Fehden oder an Naturkatastrophen als Ursachen dieser Zerstörungen glauben, müssen zunächst einmal folgende Dinge glaubhaft erklären: Auch in früheren Zeitläuften der mykenischen Periode gab es Zerstörungen durch Krieg oder durch Naturgewalten; immer aber hat man die Paläste wieder aufgebaut, und der Kulturhabitus zeigt keinen Bruch. Warum also baute man nun, nach den Zerstörungen der Paläste am Ende von SH III B, die palatialen Anlagen nicht mehr auf? Warum hört die Palastkultur auf? Bewirken Katastrophen normaler Weise, daß man die Schriftlichkeit aufgibt? Warum ändern sich im Frühen SH IIIC die Siedlungsstrukturen? Wieso unterscheidet sich in Tiryns in dieser Zeit die Siedlungsorganisation der Unterburg von jener der Außensiedlung? $?^{37}$ Wie soll man schließlich die Mengen der handgemachten Fremdware erklären, die nach den Katastrophen in den Siedlungsschichten des Frühen IIIC aufscheinen?

Man nehme es mir nicht übel: kaum einer der Erklärungsversuche, die eine Fremdeinwirkung ausschließen, vermag zu überzeugen. Damit soll nicht gesagt sein, daß Naturkatastrophen (Erdbeben) da und dort nicht tatsächlich Vorschub einer Fremderoberung geleistet hätten. Aber als alleinige Ursachen dieses totalen Bruches mit der jahrhundertealten palatialen Tradition des Mykenischen reichten sie wohl nicht aus. Dasselbe gilt für innermykenische Fehden oder für wirtschaftliche Krisen. Wie alles in der Historie, werden auch die Katastrophen der mykenischen Paläste keine monolineare Kausalität haben. Doch eine der Komponenten bestand m. E. sicher im Angriff fremder Völkerschaften von Bereichen außerhalb der mykenischen Welt, vorwiegend wohl aus dem Adriaraum.

Ob und wieweit diese Gruppen mit den „Seevölkern“ zusammengebracht werden können, ist schwer zu sagen. Sicher nicht beteiligt am Seevölkersturm auf Ägypten waren jene Fremdlinge, die im griechischen Bereich sich angesiedelt hatten. Manche von diesen, wie die fremden Zuwanderer von Aigeira, ließen sich auf neuem Boden nieder und waren offenbar bestrebt, neues, eigenes Siedlungsland zu finden ${ }^{38}$. $\mathrm{Da}$ aber einige der Seevölkernamen in den ägyptischen Quellen und in Ugarit, wie wir gehört haben, in den adriatischen Raum weisen, sollte zu denken geben.

37 K. KILIAN, AA 1979, $409 \mathrm{f}$.

${ }^{38}$ Zur Siedlungssituation von Aigeira vgl. Fremde Zuwanderer, 1.2, 1.4.1.1. 


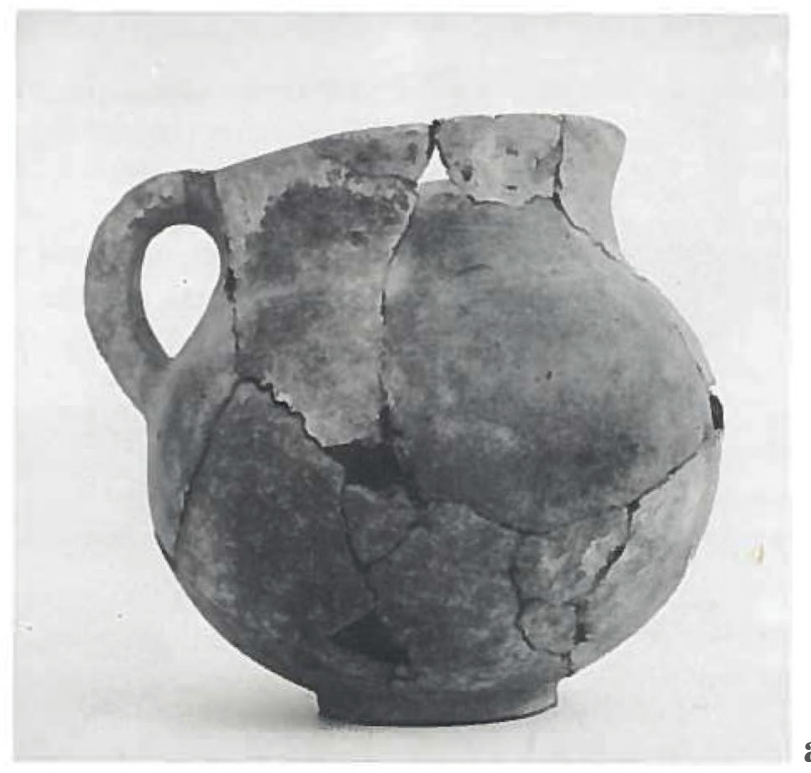

a)
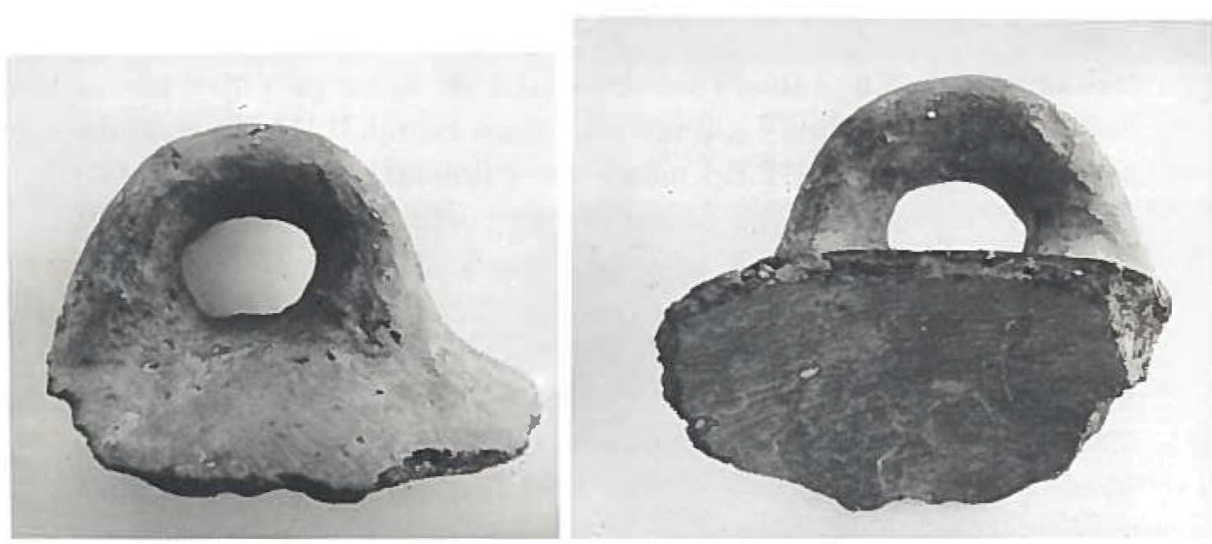

b)

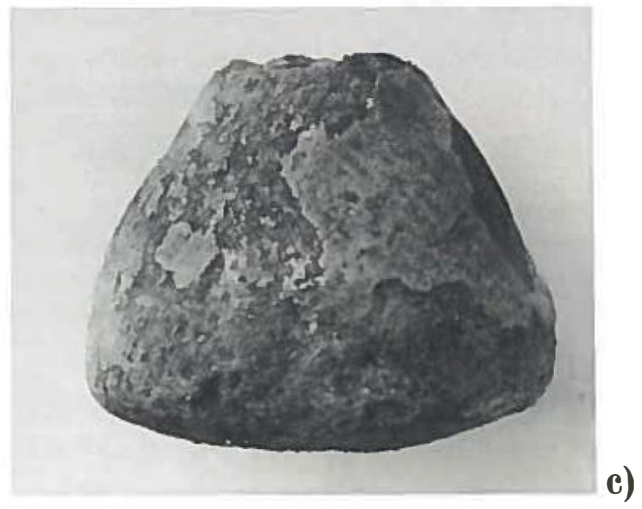

Abb. 1 : „Handmade Burnished Ware“ aus Aigeira

c) Spinnwirtel 


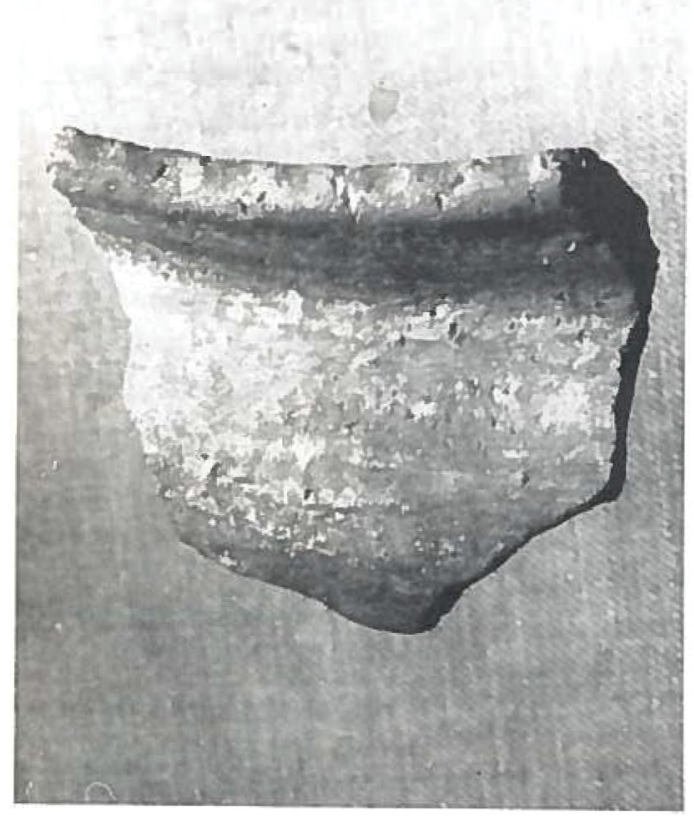

a)

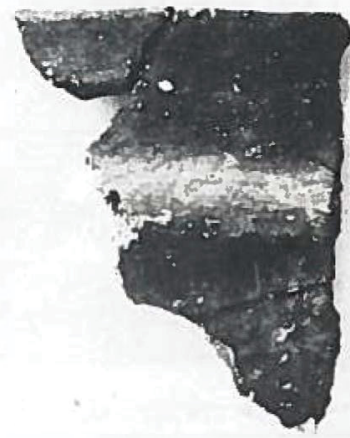

b)

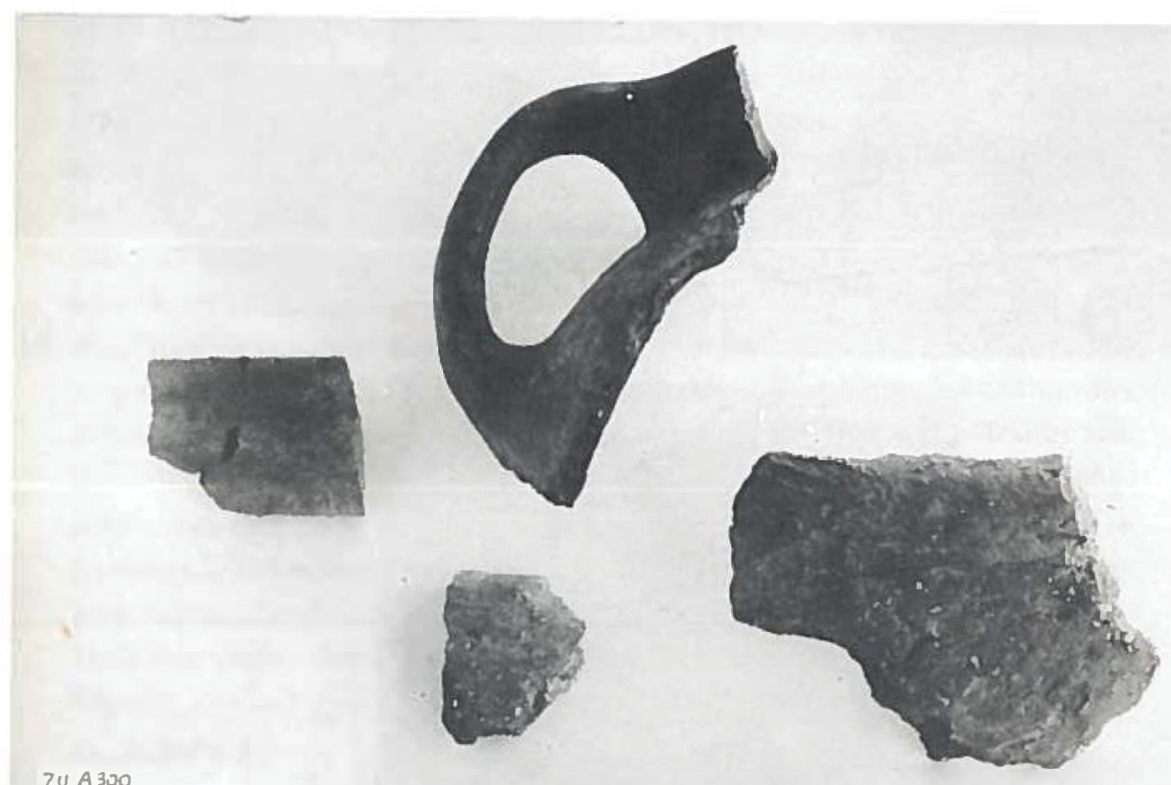

c)

Abb. 2: ..Handmade Burnished Ware" aus Aigeira

a) Tassenfragment mit Fingertupfendekor auf der Lippe -- b) Fragment von carinierter Tasse - c) Verschiedene Fragmente 


\section{Diskussion im Anschluss an den Vortrag Deger-Jalkotzy}

\section{Bittel:}

Wie ist denn eigentlich die Fundsituation dieser Stücke aus Korakou, die aus der Grabung BlEgEN stammen?

Jalkotzy:

Sie wurden in SH III-Kontexten gefunden. Die drei Ganzgefäße nahm BLEGEN in seine Grabungspublikation auf [Korakou, Abb. 104 und 105], unter der Bezeichnung "grobe Haushaltsgefäße“. Wohl vermerkte der Ausgräber, daß es handgemachte Gefäße seien, doch nahm er nicht wahr, daß es sich tatsächlich um eine eigene Keramikgattung handelt, die sich von der gewöhnlichen mykenischen Haushaltsware stark unterscheidet. Dies war die Entdeckung von J. RUTT'ER, als er viel später in Korinth das spätmykenische Material für seine Dissertation durcharbeitete. Die Eigenart dieser keramischen Gattung hatten wohl auch schon die Bearbeiter der Funde von Mykene erkannt, wo sie in SH III C datiert wurde. Es war das Verdienst RutTers, diese Gattung und ihre Datierung ins Frühe SH III C als überregionales Phänomen in Griechenland definiert und ihre historische Tragweite erfaßt zu haben. Durch seinen Artikel im AJA 79 (1975) hat RuTTER die Aufmerksamkeit der Ausgräber auf dieses Phänomen gelenkt. Als beispielsweise in Aigeira im Herbst desselben Jahres 1975 die ersten Fragmente der handgemachten Fremdkeramik in SH III C-Kontexten gefunden wurden, wußten wir sofort, womit wir es zu tun hatten. Früher wurde diese Keramikgattung häufig als MH oder FH klassifiziert.

Ergänzend möchte ich erwähnen, daß Oberflächenfunde von Seliana, einem Ort im Hinterland von Aigeira, frühhelladische Keramik erbrachten. Der Vergleich zwischen diesen Stücken und der Handmade Burnished Ware von Aigeira zeigt ganz deutlich, wie sehr sich diese beiden Keramiksorten voneinander unterscheiden.

\section{Schachermeyr:}

Es gibt, wie ich glaube, eine ganze Reihe von barbarischen Gattungen, die früher oder später gelegentlich in den ägäischen Bereich Eingang gefunden haben. Man muß sich davor hüten, sie als ein allzu gemeinsames Phänomen anzusehen. Eine der bedeutsamsten ist 
allerdings diejenige Ware, welche RUTTER erstmalig als eigene Gattung erkannt hat und die jetzt von Frau Jalkotzy in so vortrefflicher Weise behandelt wird. Ich möchte dabei vor allem auf die FingertupfenLeisten und auch auf die Gefäßformen Gewicht legen. Die Oberflächenbehandlung allein ergibt insofern weit schwerer ein Kriterium, da eine gleichartige Behandlung wohl auch bei anderen Gattungen vorkommen könnte.

Als andere Gattungen möchte ich die mit eingeritzten Tannenbaum-Mustern ansehen, die u. a. auch in Medeon vorkommt, dann allerdings erst zur Zeit des Submykenischen und der Zwischenkeramik - die Lederbeutelware (verstreut in ganz Griechenland). Eine Barbarische Fremdware mit eingeritzten Zinnenmäandern gibt es $\mathrm{m}$. W. im Museum von Olympia. Sie ist sicher aus Italien gekommen.

Unsere Fingertupfen-Ware hat als ein besonderes Charakteristikum, daß sie zwar einstmals im Frühhelladikum schon von Norden her aufgetreten ist, dann aber erst wieder im ausgehenden Late III B bzw. in Early III C Griechenland erneut erreichte. Mit der Ostbalkanischen Buckelkeramik hat sie nichts zu tun.

\section{Themelis:}

Sie haben Fundorte in Epirus erwähnt, Elaphotopos, Mazaraki, Kastritsa usw., deren Material mit Ihren Funden von Aigeira gleichzeitig sei. Aber Sie haben nicht Vitsa genannt, wo dieses Material sehr viel später auftritt, im 8. Jh., 9. Jh. Was hat dieses Material zu tun mit Ihrer Ware und mit den Gefäßen aus Elaphotopos, Mazaraki etc. ? Ist es eine Entwicklung von diesen Gefäßen her? Oder ist es für Epirus etwas Neues? Es sind handgemachte Gefäße mit Ritzlinien, also Dekorationselemente, die auch auf Ihrer Keramik auftauchen.

\section{Jalkotzy :}

Ich kenne nicht viel von dem Material aus Vitsa. Doch wenn ich mich recht entsinne, soll dort sehr wenig Keramik gefunden worden sein, die sich mit der hier behandelten Gattung vergleichen ließe. Die Materialvorlage von Frau VокотороuLOU soll aber bald erscheinen, und dann läßt sich Genaueres über diesen Punkt sagen.

[Anm. d. Hrsg.: Der folgende Teil dieses Diskussionsbeitrages ist eine nachträgliche Erweiterung.] 
Zusätzlich möchte ich auf ein Problem mit der epirotischen Grobware zu sprechen kommen, das darin liegt, daß sie chronologisch praktisch in der Luft hängt. Lediglich für das Material von Elaphotopos ergibt sich ein datierender Anhaltspunkt dadurch, daß es in der Nähe der Kistengräber von der Wende des 13. zum 12. Jh. v. Chr. lag. Die Ausgräberin, Frau Vокотороulou, nimmt daher an, daß auch diese Stücke in diese Zeit gehören, wobei mir diese Datierung besonders für jene Stücke einleuchtet, die sie der keramischen Kategorie III nach DAKaris [dazu Prakt. 1951, $177 \mathrm{ff}$.] zuordnet: Ephem. 1969, 184 und Tfl. $25 \beta, \gamma$. Sie haben Parallelen im früheisenzeitlichen Albanien und lassen sich in manchem auch mit Stücken aus Vergina vergleichen. Zur Buckelkeramik, wie N. HAMmOND dies vorgeschlagen hat, gibt es keine Beziehungen. Auch zu unserer $\mathrm{HBW}$ aus Aigeira sehe ich keine direkten Bezüge; typologische Ähnlichkeiten bestehen allerdings insofern, als sich diese epirotische Gattung durch gelegentliche Tendenzen zu Carinierung und Riefung in den Rahmen der früheisenzeitlichen Keramik des illyrischen Raumes stellt. Problematisch an der sog. Kategorie III der epirotischen Grobkeramik ist freilich, daß innerhalb dieser Gruppe das Material nicht homogen erscheint. So wirken etwa die Prakt. 1952, 369f. und Tfl. 5f. veröffentlichten, stratigraphisch nicht gesicherten Funde aus Kastritsa altertümlicher als die erwähnten aus Elaphotopos. Man fragt sich aber, ob die Unterschiede nur chronologisch sind: kann man diese diversen Stücke überhaupt typologisch zusammenstellen?

Die epirotischen Kategorien I und II, also Gefäße mit Fingertupfenleisten und anderem plastischen Dekor sowie solche mit Warzenverzierung, sehe ich jetzt ebenfalls komplizierter als zur Zeit der Abfassung meiner Fremden Zuwanderer. Damals teilte ich RUTTERs Ansicht, daß hier keine Parallelen zur HBW des mykenischen Griechenland vorlägen. Später freilich sah ich im Museum von Joannina Keramikfragmente vor allem aus Kastritsa, die in Oberflächenbehandlung, Form und Verzierung tatsächlich vergleichbar unserer Fremdkeramik sind, mit Verbindungen zu Subapenninischen Keramik Italiens. Auf solche Stücke beruft sich der Ausgräber von Tiryns zurecht als Parallele zu seinem Material. Fragmente derselben Gattungen sind nun aber auch unter den Funden von Elaphotopos, die Frau Vокотороulou ja um 1200 datiert (s. oben). Innerhalb der Kategorien I und II gibt es aber m. E. Exemplare, für die meine frühere Ansicht weiterhin gilt: in ihrer mangelhaften Formgebung und Oberflächenbehandlung erscheinen sie mir wie hinterwäldlerische Spätlinge aus mittel- wenn nicht 
frühbronzezeitlicher Tradition. Sind dies einheimische Vorläufer der stilistisch besseren Stücke, die ich vorhin erwähnt habe? Auch K. A. WARDLE denkt an chronologische Unterschiede innerhalb dieser Keramikgattung. Oder sind es weniger gut gelungene Versionen einer Keramik, die ebenfalls erst um 1200 in Epirus auftritt?

Genauere typologische Untersuchungen an den epirotischen Keramikgattungen sind also ein Desiderat. Doch auch so darf man jene Stücke, die als Parallelen zu unserer HBW gelten können, m. E. nicht ohne ihren illyrischen Rahmen und nicht ohne ihre Beziehungen zur Subapenninischen Kultur sehen. Betonen möchte ich schließlich noch einmal, daß diese Parallelen generell bleiben, soweit Aigeira betroffen ist. Das Material von Aigeira kennt keine Warzenverzierungen; umgekehrt fehlen in Epirus eine ganze Reihe von Formen, die Aigeira mit Süditalien verbinden. Das aigeiratische Material allein mit Nordwestgriechenland zu verbinden, würde Einäugigkeit bedeuten.

Falls man ferner nur mit kleinräumigen Transhumance-Bewegungen rechnet, müßte man Material für Akarnanien und Aitolien belegen.

\section{Georgiev:}

Ich beschäftige mich eigentlich nicht so sehr mit diesem Problem wie Frau Jalkotzy, aber ich möchte etwas zur gewöhnlichen, zur sog. Küchenkeramik sagen. In Südbulgarien z. B. gibt es da eine Kontinuität vom Anfang der Bronzezeit bis zum Ende der Bronzezeit, sogar bis zum Anfang der Eisenzeit. Das ist eine Ware, die eher zur Kontinuitätsfrage beitragen kann. Sie synchronisiert vielleicht mit den nordpontischen Gebieten, aber von einer direkten Beeinflussung aus dem nordpontischen und rumänischen Raum würde ich doch Abstand nehmen. Einflüsse durch Makedonien sind bestimmt vorhanden. Aber weitere Einflüsse sind, glaube ich, zu weit hergeholt.

\section{Jalkotzy :}

Das ist Rutrers Hypothese. Doch dürfte es 'stimmen, daß in den genannten Gebieten diese Keramik eine längere Tradition hat als in der Ägäis.

\section{Georgiev:}

Gewöhnliche Keramik kommt aber an sehr verschiedenen Stellen 
vor, und man kann sie oft nicht datieren. Es gibt da keine feineren Unterschiede in den Henkeln, in der Technologie.

\section{Eibner:}

Die Evidenz von verschiedenen Keramikgattungen stößt in der Beurteilung auf um so mehr Schwierigkeiten, je undifferenzierter und einfacher das Material ist. Es kommt vor allem auf die Formensyntax an, neben der gediegenen Materialkenntnis.

\section{Jalkotzy :}

Die HBW kann man nicht einfach als Küchenkeramik bezeichnen. Sie hat ihre eigene Technik, Verzierung und Formensyntax, die sich von denen der mykenischen Keramik grundlegend unterscheiden, die aber an sich einen eigenen Stil ausdrücken. Sie ist also nicht undifferenziert, sondern läßt sich in ihrer Eigenart sehr wohl mit jenen Gebieten verbinden, die ich im Vortrag genannt habe. Dabei ist es aber so, daß die Rückbeziehungen von Fundort zu Fundort variieren, ja selbst an ein und demselben Fundort nach verschiedenen Richtungen laufen.

\section{Eibner:}

Die Einhenkeligkeit der Gefäßformen (Tassen, Krüge) ist im zentralen Mitteleuropa typisch, mit Tradition bis ins Endneolithikum. Dagegen bildet der Balkanraum im weitesten Sinn gerne Doppelhenkelformen aus, so daß man, zumindest im Argument, auf die Ausbreitung der Gefäßformen achten sollte.

\section{Bittel:}

Das Großraumgebiet, das wir hier vor uns haben, ist sehr beeindruckend, und da eröffnen sich Perspektiven und Bezüge, die von wirklich historischem Ausma B sind. Natürlich ist die Herstellung der Keramik abhängig von den einzelnen Tonlagern, von der Geschicklichkeit der Hände, der Aufbereitung und von der technischen Kenntnis. Trotzdem ist es, glaube ich, gut, wenn man danach strebt, die Sachen auch in die Hand zu nehmen. Abbildungen reichen of $t$ nicht aus. Ich hoffe, daß diejenigen, die Ihnen wohlwollen, dabei behilflich sind. Wenn man die Dinge in die Hand nimmt, gewinnt man ein stärkeres 
Zutrauen, ein intimeres Verhältnis. Besonders wenn man über so weitreichende Verbindungen zu urteilen hat, wäre dies sehr wichtig.

\section{Jalkotzy:}

Die Ausgrabung auf der Akropolis von Aigeira wurde während der ersten Jahre von einer Wiener Prähistorikerin durchgeführt, die eine Spezialistin für die Urnenfelderzeit im pannonischen Raum ist. Ihr kann man, glaube ich, durchaus die entsprechende Urteilsfähigkeit zutrauen. Einen anderen solchen Spezialisten, im besonderen der frühen Urnenfelderkultur, haben wir unter uns. Beide Freunde haben mir in der großzügigsten Weise Material zugänglich gemacht und durch Diskussion enorm geholfen. Schade ist nur, daß diese Materialien vielfach unpubliziert sind. Sicherlich lernt man aus diesem Material, daß von einer massiven „Urnenfelderwanderung“, wie man früher gemeint hat, nicht gesprochen werden kann. Unzweif elhaft aber gibt es Verbindungen, die in den Adriaraum ebenso wie in den Balkan führen. Die Schlüsse, die daraus zu ziehen wären, sollten wir aber eigentlich erst nach dem Vortrag von Herrn Dobesch erörtern. - Einiges der oberitalienischen Keramikfunde kenne ich durch Autopsie; durch das freundliche Entgegenkommen der Kollegen aus Trento konnte ich auch manches im Trentino Gefundene in die Hand nehmen. - Apulische subapenninische Keramik aus Privatbesitz durfte ich genau untersuchen. Wären nicht die individuellen Unterschiede der Herstellung (insbesondere was die Mineralien der Magerung anlangt), auf die Sie hingewiesen haben, müßte ich mich hüten, solche Fragmente unter die Funde aus Aigeira zu mischen; man könnte sie schwer wieder herausfinden. - Die Vergleichskeramik aus Ithaka habe ich in der Hand gehabt, jene aus Kephallenia gesehen. Letzteres gilt auch für keramische Funde aus Epirus. - Keine persönliche Erfahrung habe ich mit Materialien aus den Balkanländern. Hier darf ich mich aber auf Herrn Bouzek berufen, der diese Funde gut kennt.

\section{Kirsten:}

Der Historiker, der sich in der letzten Zeit mit Süditalien hat beschäftigen müssen, ist immer vor der Frage gestanden: Wann sind die illyrischen Sprachgebiete in Italien entstanden? Zwei verschiedene sind es: Einerseits das der Veneter am sog. Caput Adriae, das Verbindungen zu Mitteleuropa hatte, da auch die Bernsteinstraße dorthin führte; und andererseits im Süden die Gruppe der Daunier, 
Peuketier, Messapier. Wir haben in letzter Zeit Kongresse im daunischen Gebiet gehabt, wo man besonders eifrig ist, die Beziehungen über die Adria hinweg (zu Albanien) zu berücksichtigen, haben dabei die Parallelen zu analogen Erscheinungen viel später, nämlich zur slavischen Landnahme des Gargano, beobachtet, und haben. ebenfalls nie eine zeitliche Fixierung gefunden. Gehen wir ganz allgemein morphologisch vor, so steht keine Keramik Italiens der hier betrachteten KnickKeramik so nahe wie die der Gattungen der apulischen Keramik. An dieser wird der Knick freilich nur dadurch datiert, daß die Vasen geometrische Ornamente tragen, aber das besagt ja noch nicht, daß sie gleichzeitig sind mit der Entwicklung der geometrischen Stile Griechenlands (vgl. die Befunde von Sala Consilina im antiken Lucanien) - sicher reichen die Ausläufer dieser epichorischen trozzelle u. dgl. bis in hellenistische Zeit hinein (in Gräbern der Terra d'Otranto unter Stadtmauern des 2. Punischen Krieges, vgl. meine Süditalienkunde I $37 \mathrm{ff}$.). Es bleibt die Frage, ob die illyrischen Einwanderer nach der „Ferse“ Süditaliens zwischen Gargano und Kap S. Maria di Leuca schon die geometrische Keramik und die Knick-Formen mitbrachten, und wann (im 12.-10. Jh., sicher vor den ersten griechischen Kolonisten; doch kennen wir die Küsten Albaniens leider noch viel zu wenig).

\section{Hölbl:}

Ich habe mich immer gefragt, wie weit wir die Peleśet und Tekker mit diesen „Barbaren“ in Verbindung setzen könnten; daher habe ich auch in meinem Referat mit besonderem Grund die Stelle im Papyrus Harris herausgestrichen, wo nur die Denjen (Danaoi) die eigentlichen Inselbewohner sind, so daß vielleicht eine gewi. se Kenntnis bis nach Ägypten gelangt ist, nach der die anderen Stämme keine ägäischen Inselbewohner wären.

\section{Lehmann:}

Ich darf hier vielleicht noch an mögliche historische Zusammenhänge erinnern, die allerdings nur in einem Komplex antiker Orts- und Ethnien-Namen im adriatisch-balkanischen und italischen Raum andeutungsweise greifbar sind:

1. die räumliche Nähe zwischen Toponymen im adriatisch-nordwestbalkanischen Bereich, die mit dem Ethnikon der *Palaistai-Philister wahrscheinlich in Zusammenhang stehen (Palaiste an den alkroke- 
raunischen Bergen; Palaistinos als Bezeichnung für den Oberlauf des Strymon), und dem Gebiet der illyrischen Hylleer um Korfu; 2. die enge Verbindung zwischen den adriatischen Hylleern/Hyllinoi der sog. hylleischen Chersones und einem Gemeinwesen von Siculi einerseits, sowie andererseits der Siculotae und Sardeates weiter südlich ebenfalls an der adriatischen Küste; 3 . die bemerkenswerte und durchaus analoge Streuung des Teukrer-Ethnikons vom adriatisch-balkanischen Raum über die Ägäiswelt hin bis zu Levanteküste. Schließlich lassen sich zu diesen Kombinationen durchaus enge Entsprechungen auch an der West- und Ostküste Italiens aufzeigen (zwischen Šardana-Sardones, Sikelern und Turša-Tyrhsenern). Die beigefügte Kartenskizze möge dies veranschaulichen. 


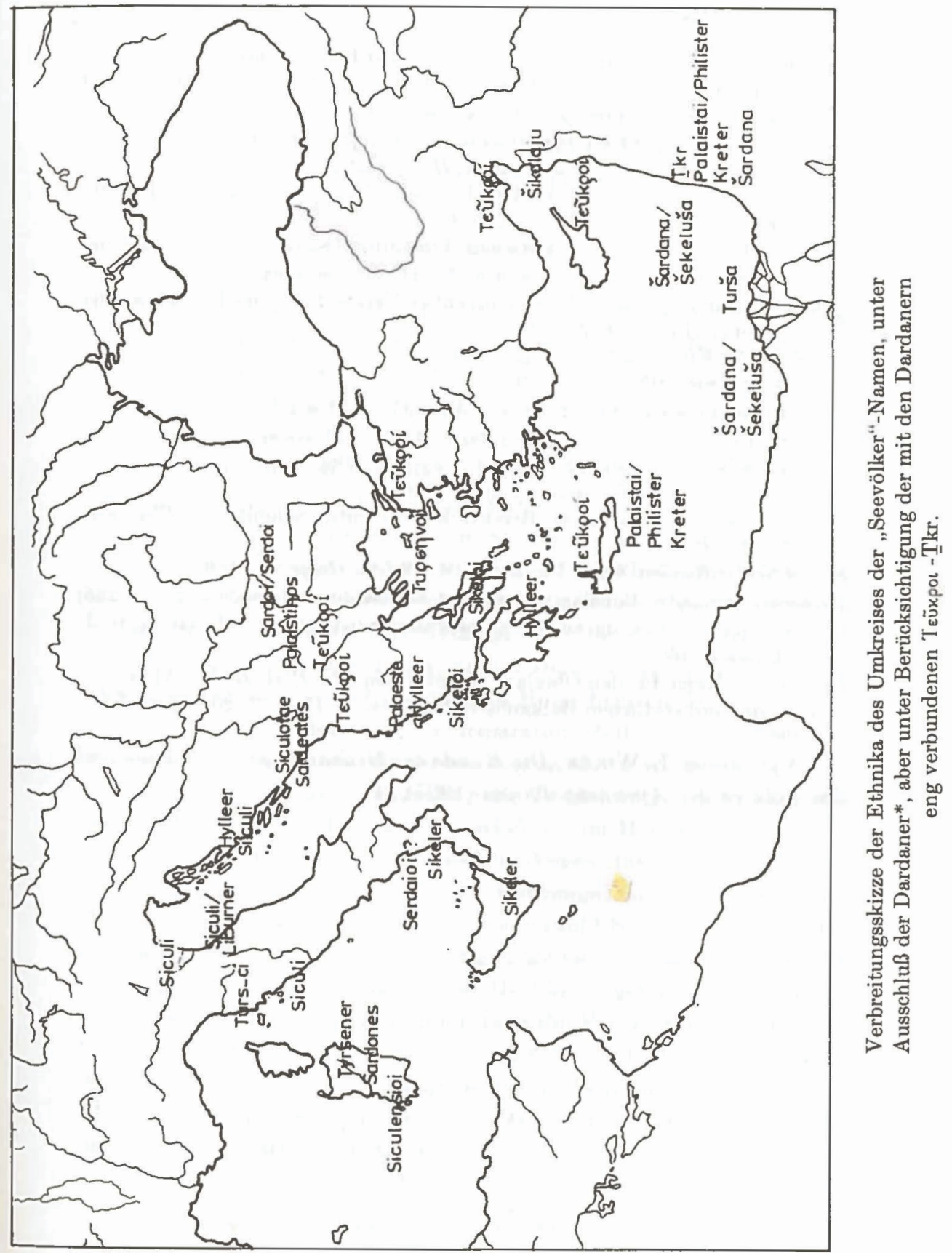


Kommentar zur Skizze:

*Dardaner und Danu-Danuna- $\Delta \alpha v \alpha \overline{5}$ oi (hier nicht berücksichtigt): Belege und Expansionsgebiete vom Morava-Tal über Apulien bis zur Troas und nach Kleinasien (drdny in den Kadeš-Texten).

Sikeler: in Latium (Antiochos v. Syrakus b. Dion. Hal. 1, 73, 4)

in Bruttium (Thuk. 6, 2, 5; Dion. Hal. 1, 22)

in Picenum (Plin. n.h. 3, 110-112; allgemein Dion. Hal. 2, 1, 1; 1, 9)

in Sardinien (Ptolem. Geogr. 3, 3, 6)

Siculi: dalmatin. Küstenort zwischen Trogir und Salona, d. h. südlich der hylleischen Halbinsel (Plin. n.h. 3, 141; Ptolem. Geogr. 2, 16)

Siculotae: Stamm im Bereich des conventus Naronensis / um Narona an der Neretva (Plin. n.h. 3, 143)

Siculi Sikeler-Ethnikon in der Ägäiswelt:

Akarnanien (Paus. 1, 28, 3)

Attika (Paus. 8, 11, 12; Dion. Chrys. 17, 17 Arn.)

im Umkreis der Peloponnes (Steph. Byz. s. v. $\Sigma\llcorner x \in \lambda i x$.)

im euböischen Sund (Schol. Eur. Phoeniss. 208)

Serdaioi/Serdaner: Inschr. aus Olympia; Münzen

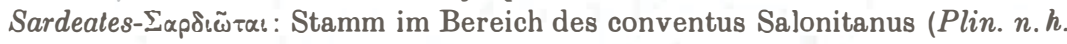
3, 142; Ptolem. 2, 17)

Sardoi/Serdoi/Serdica: Cass. Dio 51, 24, 4; Ptolem. Geogr. 3, $11,8$.

Tyrhsener: als „alte" Bevölkerungsschicht auf Sardinien (Strabo 5, 2, 7 p. 225)

Palaeste: südl. v. Oricum an der akrokeraun. Küste (Caes. bell. civ. 3, 6, 3;

Lucan 5, 460)

Palaistinos: Name für den Oberlauf des Strymon (Ps.-Plut. de fluv. 11, 1)

TEũxpo: : im nordwestlichen Balkanbereich (Herod. 5, 13, 2; 7, 20; 75, 2)

Vgl. weiter E. WIKÉn, Die Kunde der Hellenen von dem Lande und den Völkern der Apenninhalbinsel (1937) $21 \mathrm{f}$. 